
| |

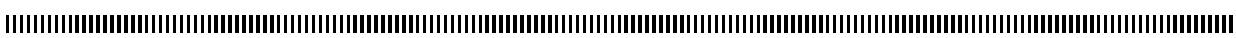

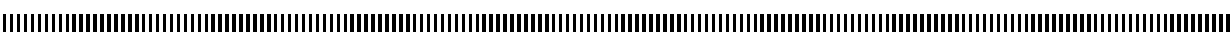

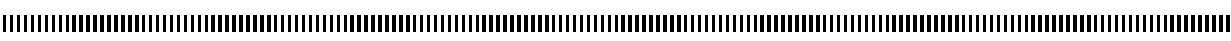
| |

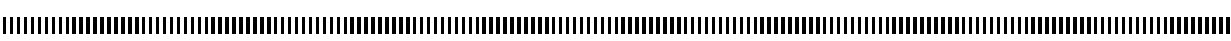

\title{
Système d'Information Intégré Adaptatif sous Web pour la gestion et la modélisation des ressources hydriques ${ }^{1}$
}

\author{
F. El Dabaghia - M. Bechchiab - H. Henineac \\ a INRIA Rocquencourt B.P. 105, 78153, Le Chesnay Cedex \\ b Ecole Mohamadia d'Ingénieurs, B.P. 765, Rabat, Maroc \\ c Ecole Nationale Polytechnique, B.P 10 Avenue Hassen Badi, El Harrach, Alger, Algérie.
}

|

RÉSUMÉ. Ce travail présente le Système d'Information Intégré Adaptatif sous Web (SIIAW) pour la gestion des ressources hydriques. Le SIIAW est une extension naturelle du S2IWV-WADI vers une architechture adaptative et personnalisée en fonction de l'utilisateur et du contexte d'utilisation incluant: un système de recherche et d'indexation, des codes de simulations, un SIG et des mailleurs ainsi que des visualiseurs graphiques. Ce SIIAW permet ainsi d'une part de manipuler de manière automatique les données des simulateurs et d'autre part d'exploiter et de caler éventuellement en temps réel les résultats de ces simulations en les corroborant avec des paramètres extraits ou identifiés.

ABSTRACT. This work presents the Adaptative Integrated Information System under Web (AIISW) for the management of hydrous resources. The AlISW is a natural extension of the IISW-WVADI towards an adaptative and customised architecture according to the user profile or the context of use including : a research and indexation engine system, simulators, a GIS and meshing editors as well as graphic viewers. This AlISW allows on the one hand to manage in an automatic way data of simulators and on the other hand to exploit and calibrate eventually in real time the results of these simulations by corroborating them with extracted or identified parameters.

MOTS-CLÉS : Système d'Information Intégré, WEB, moteur de recherche et d'indexation, Profil utilisateur, Ontologie du domaine, thésaurus, Substitut de document, ressources hydriques, simulateurs numériques.

KEYWORDS : Integrated Information System, WEB, search and indexing engine, User profile, Ontology of the field, thesaurus, Substitute of document, hydrous resources, numerical simulators.

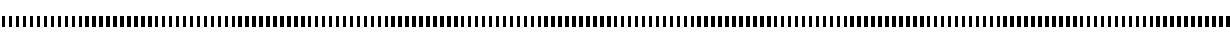

1. Ce travail a bénéficié du support du projet euro-méditerranéen WADI, des programmes d'actions Intégrées CMIFM MA/01/03., CMEP 01 MDU 529 et PLATON 05572UB.

Volume 5 - 2006, pages 52 à 64 - ARIMA



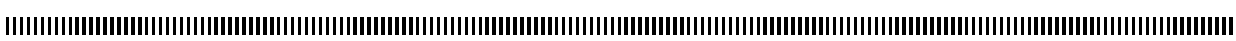
| |

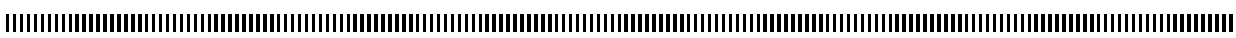

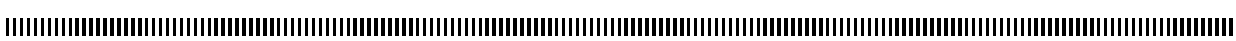

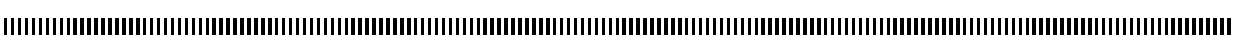
| |

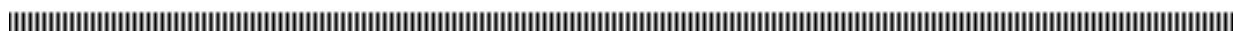




\section{Problématique}

La problématique de l'eau constitue un domaine prioritaire tant au niveau des instances nationales, régionales qu'internationales. En particulier, dans la zone méditerranéenne où la demande croissante en eau est associée à une diminution de cette ressource, son utilisation intensive ainsi que les divers risques (pollution, inondation, etc.) nécessitent une gestion rationnelle et optimale de celle-ci. Dans ce contexte alarmant, ces risques inhérents nous interpellent en priorité pour une meilleure compréhension du processus des ressources en eau, caractérisé par l'interaction de plusieurs phénomènes physiques complexes en soi et du rôle intrinsèque et vital de l'eau, ressource devant être durable et renouvelable, dans les besoins de la société. Qu'il s'agisse de préserver l'eau pour l'irrigation, d'aménager un bassin versant, de construire un barrage pour le stockage de l'eau ou pour diminuer les risques d'inondation ou de vouloir oxygéner un lac pour éliminer les problèmes d'eutrophisation, il est clair que n'importe quelle stratégie pour développer une solution durable, pérenne et évolutive, même partielle, pour cette problématique, doit être basée sur une approche qui considère les relations de cause à effet et qui évalue de manière systématique les diverses solutions.

Ce travail s'inscrit dans cette logique avec pour objectif la réalisation d'un Système d'Information Intégré Adaptatif sous Web (SIIAW) [8] [9] [21], un élément d'aide dans la chaîne de prise de décision permettant la simulation numérique, le traitement d'images et la gestion des données hétérogènes pour la prédiction et l'estimation de l'évaluation et de l'évolution des phénomènes hydriques.

\section{S2IW : caractéristiques et architecture}

Le S2IW est une application WEB de type client/Serveur développée sous Apache-Tomcat [24]. Son architecture logicielle (Figure 1) est basée sur la possibilité d'intégration d'éléments logiciels (composants logiciels) provenant de sources différentes et par la prise en compte des systèmes informatiques existants. Il implique l'intégration et l'interactivité entre des outils très divers mais complémentaires. Au lieu d'un énorme outil multifonction, il est basé sur la sélection ou le développement d'un ensemble de technologies permettant, par assemblage, la création d'un système d'information intégré configurable et dynamique, adapté si possible, aux besoins spécifiques des utilisateurs et aux moyens de calculs disponibles. Les principaux composants du S2IW sont:

- SIG: Le système d'information géographique [14] [17] dédié à l'acquisition, le stockage, la manipulation, l'analyse et la visualisation de données qui sont référencées spatialement. Le SIG est généralement à même de combiner indifféremment des fichiers Rasters (Images), des fichiers vecteurs (objets) et des fichiers bases de

ARIMA - numéro spécial TAM TAM'05 
données.

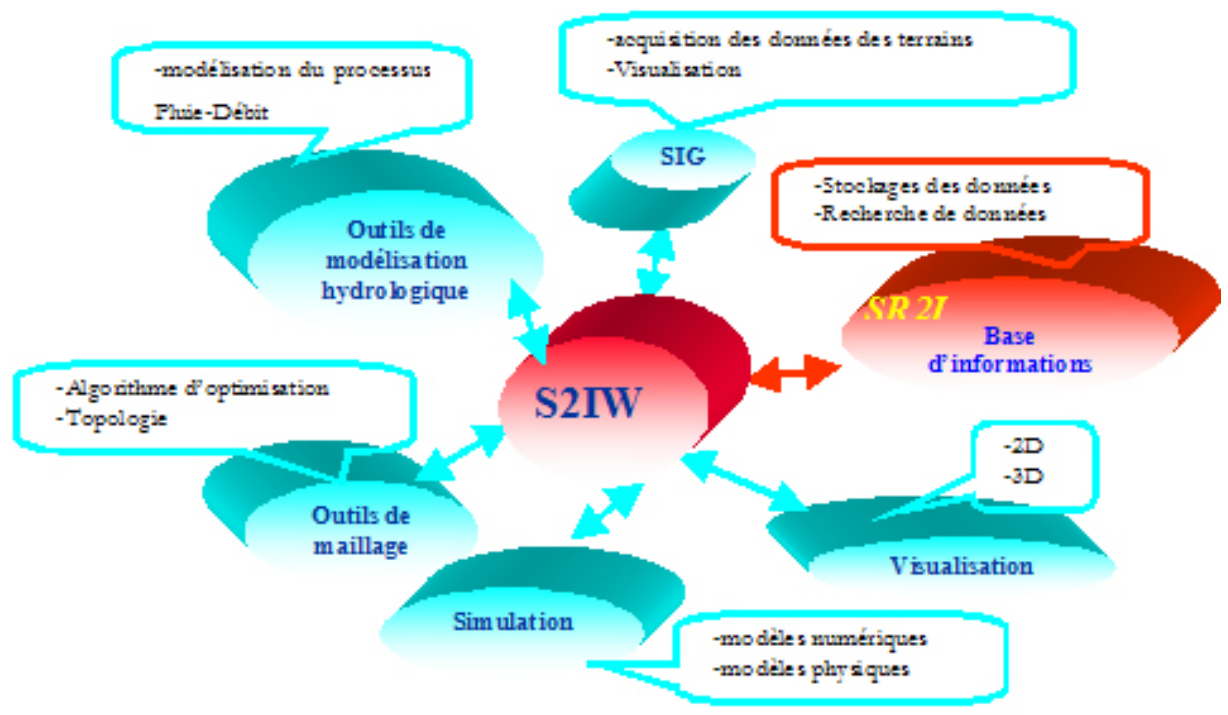

Figure 1. Architecture logicielle du S2IW

- Outils de modélisation hydrologique: Ils proposent une variété de techniques pour modéliser le ruissellement, les infiltrations, l'écoulement en rivière ou encore la répartition des pluies en vue d'une modélisation du processus pluie-débit dans un bassin versant. Notre choix s'est porté sur le package HEC [15] [16].

- Logiciel de maillage 2D/3D: Ces logiciels sont nécessaires pour la création de maillage discrétisant les domaines géographiques où a lieu le phénomène à simuler en un ensemble de simplexes géométriques. Les éditeurs de maillage utilisés sont, tous développés à l'INRIA, pour le 2D, EMC2 et YAMS, et pour le 3D, YAMS et GHS3D.

- Simulateurs numériques: La simulation numérique a pour objectif de résoudre les modèles physico-mathématiques (Equations de Navier-Stokes, Euler et SaintVenant) décrivant les phénomènes hydrauliques tels que l'eutrophisation [1] [2], l'aménagement des bassins versants [16] [17] et la prévention contre les risques d'inondation [10] [11] [12] [23]. Les codes de calcul sont basés sur des modèles numériques utilisant essentiellemnt la méthode des caractéristiques pour l'approximation en temps et les éléments finis pour la discrétisation spatiale, nécessitant un temps de calcul important. Cette lourdeur du code est justifiée par la grande taille des matrices à résoudre. 
- Logiciel de visualisation: Les logiciels de visualisation des résultats numériques devraient permettre de définir les paramètres de la visualisation $2 \mathrm{D} / 3 \mathrm{D}$, de lire différents types de base de données topologiques et de solutions associées et de les présenter graphiquement avec des possibilités de définir des coupes, d'extraire des courbes, de tracer des isovaleurs et d'effectuer des variantes graphiques de présentation. Ces logiciels sont développés sous Fortran et $\mathrm{C}$ avec des librairies graphiques du type X11, OpenGL sous Unix et OpenGL et SDK sous MS-Windows. On utilise principalement VISU et MEDIT (développés à l'INRIA) et SMS8.1 (en association avec le code FESWMS).

- Base d'informations: Elle constitue une pierre angulaire du S2IW. En effet, l'efficacité d'un tel système intégré est freinée entre autres par la difficulté de recherche, le volume des informations écrites et la redondance inévitable, la compréhension des informations et la pertinence de l'information [6].

L'architecture du S2IW WADI est basée sur la répartition des traitements et des données entre des postes clients et un serveur (Figure 2). Les postes clients préparent et soumettent des requêtes au serveur (hydre.inria.fr), une machine Unix assez puissante en terme de mémoire et de capacités d'entrée-sortie, qui leur fournit des services et qui a un accès direct à une machine parallèle.

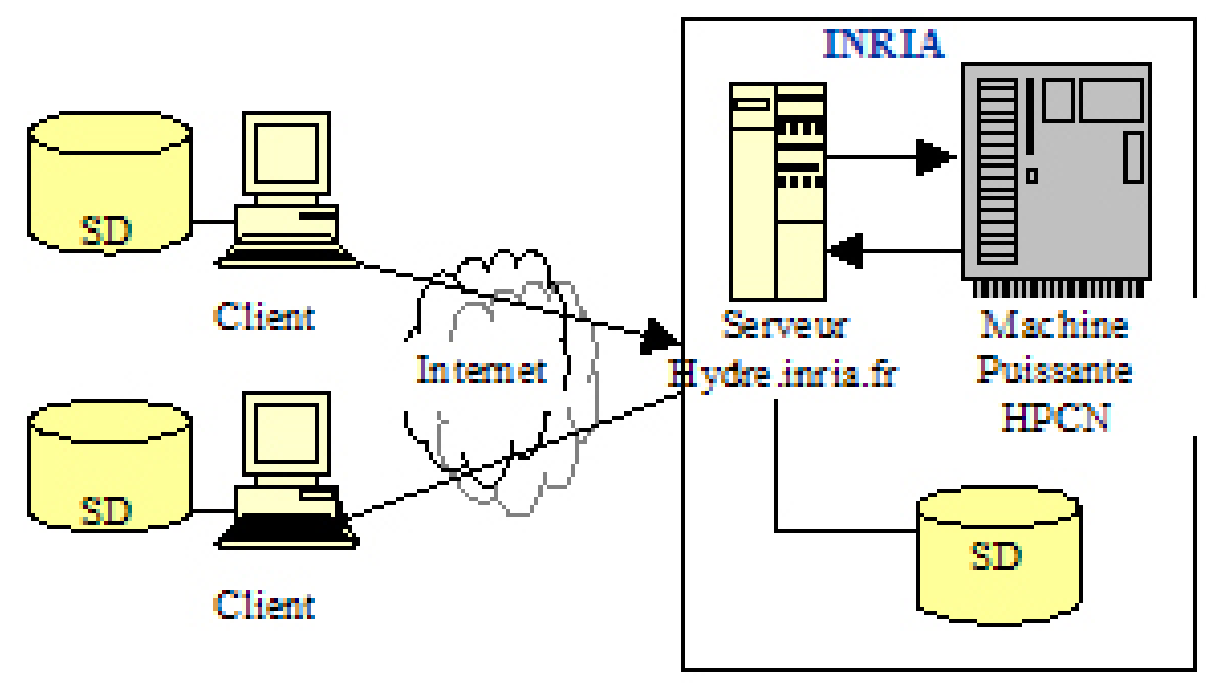

Figure 2. Architecture Client/Serveur du S2IW WADI

Cette plate-forme permet entre autres une navigation inter-application, une coopération entre logiciels et leur utilisation conviviale sur le poste client et/ou serveur, une coopération entre programmes en tant qu'environnement de dévelop- 
pement, et un accès aux ressources matérielles et logicielles distantes (machine parallèle réelle ou virtuelle, données, l'appel de procédures à distance telle l'exécution de codes lourds sur le serveur UNIX, stockage, etc ).

Ce S2IW permet ainsi d'une part d'extraire, de préparer, de manipuler, de gérer et d'analyser de manière automatique les informations nécessaires aux modèles de simulation et d'autre part d'analyser, d'exploiter, d'interpréter, de visualiser, de gérer, de communiquer, de vérifier et de caler éventuellement en temps réel les résultats de ces simulations en les corroborant avec des paramètres extraits ou identifiés.

Le développement de S2IW WADI a nécessité l'utilisation de plusieurs outils de travail : des langages de programmation, des logiciels spécifiques, des outils de gestion, etc. Le choix de ces outils est justifié par le fait que le S2IW doit être à la fois facile à gérer, portable, efficace et indépendant de tout SGBD. Ces principaux outils sont XML, JAVA et JSP. De ce fait, le système est tout à fait générique et sa transposition vers d'autres domaines d'application, voisins ou connexes, est immédiate : l'aménagement du territoire, l'aménagement côtier (ressources marines, etc..), la gestion et la modélisation des ressources énergétiques, la qualité de l'air, la météorologie, l'agriculture, l'environnement de manière générale, etc.

\section{Personnalisation}

\subsection{Du S2IW vers S2IAW : Motivation et architecture}

Une des principales limitations de ce système réside dans le fait qu'il fournit les mêmes interfaces et les mêmes fonctionnalités à des utilisateurs ayant des buts et des connaissances du sujet largement différents [13] [22]. L'approche de personnalisation constitue une extension naturelle ou enrichie de l'approche traditionnelle dans le développement de systèmes adaptatifs. Ces systèmes établissent un modèle de buts, de préférences et des connaissances de chaque utilisateur, et emploient ce modèle dans l'interaction avec l'utilisateur afin de s'adapter à ces besoins. L'adaptation du S2IW [8] [21] peut en assurer une utilisation plus complète en expliquant la façon d'atteindre un but ou en présentant des façons différentes et plus efficaces pour réaliser une tâche. La tâche n'est pas facile vue l'hétérogénéité et la distributivité des données manipulées par l'utilisateur et qui constituent une majeure partie de ces systèmes. En outre, dans le domaine des sciences de l'eau, beaucoup de modèles numériques très puissants dans l'aide à la décision, deviennent quasiinutilisables à cause de la non maîtrise des données de base qui servent comme entrées au système. De la même manière, les sorties des modèles peuvent être mal exploitées à cause de l'inadéquation des outils de visualisation et de traitement. De ce fait, on ne peut pas parler de personnalisation indépendamment du contexte ou du problème traité permettant de construire toute la connaissance d'un do- 
maine. De plus, dans ce domaine d'application il subsiste un besoin considérable en flexibilité, à la fois dans la représentation et la manipulation des données, qui sont caractérisées par l'absence de structure fixe et rigide. La mise en place d'une ontologie du domaine est indispensable et le couplage profil-document via le SRIP sera la clé de réussite du S2IAW en répondant à ces contraintes.

\subsection{Couplage Utilisateur-Document}

Les documents présents dans l'entrepôt de données ne sont pas statiques et consultés passivement, mais ils sont souvent renvoyés à la demande (recherche) où leur consultation implique une participation active de l'utilisateur (instrumentation du document, par exemple hypertexte). Ce dernier point rend donc importante la notion de personnalisation dynamique de ces documents, afin de faciliter leur consultation en tirant avantage d'une modélisation basée essentiellement sur une combinaison utilisateur et contexte d'utilisation [7]. Une recherche d'information ne vise jamais à obtenir des renseignements pour eux-mêmes; elle est faite en vue de leur exploitation dans des conditions pas toujours précises. Il faut donc que ces conditions soient connues ou au moins limitées. En particulier, il convient de savoir entre autres : Qui est le demandeur? Quelle utilisation compte-t-il faire des informations? De quel délai dispose-t-il? Quels documents connaît-il déjà sur la question et, d'une manière générale, ce qu'il sait déjà sur le sujet? Sous quelle forme, préfêre-t-il obtenir les informations? etc.

La formulation des questions par les utilisateurs risque d'être imprécise ou ambiguë à plus d'un titre. D'abord au niveau de la description du sujet, qui peut être trop large ou trop restreinte; ensuite au niveau de l'utilisation envisagée des informations recueillies. Le même sujet peut être traité différemment par des documents de différents types, dont chacun peut être mieux adapté à un contexte d'utilisation qu'à un autre. Par exemple, un document résumant les principales orientations d'un plan de protection et d'évacuation des populations dans une zone géographique sujette à un risque d'inondation peut en donner une vue d'ensemble pour la lutte contre ce phénomène, mais ne pourra pas permettre d'entamer un travail spécifique d'analyse de l'onde de crue causant cette inondation. Il faudrait dans ce cas le (les) document(s) relatif(s) à l'onde de crue et à l'inondation. Un autre exemple, après avoir établi une bibliographie d'une centaine de références rechercher les documents correspondants n'a guère d'utilité quand le demandeur doit produire une note de synthèse sur le sujet en un temps très court. Autrement dit, la question la plus fréquente : Quelles informations avez-vous sur tel sujet? " devrait être formulée par une phrase du type : Avez-vous sur tel sujet, tel type d'information me permettant de faire tel travail, dans telles conditions? $*$.

Dans un premier temps, cela nous amène à la notion de profil d'utilisateur classique tel qu'il est défini en bibliothéconomie : une équation de recherche (ensemble structuré de descripteurs), exprimant les informations que l'utilisateur désire recevoir 
régulièrement d'un service de diffusion sélective de l'information (DSI). Le plus souvent, son profil est construit par un spécialiste de l'information, qui procède d'abord à un entretien détaillé avec l'utilisateur et à des essais qu'il soumet à son appréciation pour des modifications, au fur et à mesure que ses centres d'intérêt évoluent.

Les points d'accès ou de recherche sont les diverses caractéristiques d'une information à partir desquels peuvent être opérées à la fois, la recherche et la sélection dans un entrepôt de données, en général accessible via un index ou mieux encore un thésaurus. Ils sont exprimés par l'utilisateur dans sa question, par les indications qu'il donne sur le sujet, les dates, le type de document recherché, l'auteur, le volume, l'accessibilité, etc. Ainsi, il est tout à fait logique que l'étape qui devrait suivre, associerait complètement un profil utilisateur à un contexte d'utilisation à travers une (ou partie) ontologie dynamique du domaine applicatif; celle-ci peut être vue comme un sous ensemble de descripteurs issus de thésaurus, définis et classés par ordre d'importance, mais surtout liés entre eux par des relations cohérentes [3] [19] [20]. Associer dans une description de ressources, une caractérisation de ces dernières avec celle du profil de l'utilisateur entraînera nécessairement un système d'interrogation à relance où la première interrogation ne constitue en ellemême qu'une ouverture de session interactive avec le système, avec qui s'ensuivra un dialogue itératif avec retour de pertinence [25] pouvant générer une nouvelle ontologie et la cataloguer pour une utilisation future.

\subsubsection{Ontologie du domaine}

Modéliser un domaine consiste à choisir les formules vraies et utiles dans celuici. Pour cela, il faudra déterminer les termes primitifs de modélisation du domaine et leur signification. Une ontologie fonctionne comme un cadre théorique du domaine construit en fonction du problème traité. Le processus de modélisation décrit ici est fondé sur cette dernière définition. Nous construisons l'ontologie en nous appuyant sur les connaissances présentes dans le corpus et des connaissances externes au corpus (experts). La définition de l'ontologie initiale [4] doit être réalisée avec des experts du domaine ayant une bonne connaissance du corpus et sachant exprimer la nature des informations à extraire. Cette phase aboutit à la description [5] d'un ensemble de hiérarchies de concepts constituant une première sous-ontologie (ontologie initiale). Cette ontologie met en jeu des relations argumentatives entre concepts (un concept est lié à un autre car il en est un argument). Dans chaque relation argumentative, toute valeur d'un argument est une instance du concept correspondant à cet argument. Nous présentons ci-après (Figure 3) un exemple hiérarchique d'ontologie préliminaire.

A titre d'exemple, un * profil de numéricien » interagissant avec le S2IAW à partir de cette ontologie et intéressé par les méthodes d'approximation adoptés dans les simulateurs numériques des crues, se verrait aboutir après «échange à relance * à une ontologie modifiée contenant des documents plus descriptifs des méthodes 

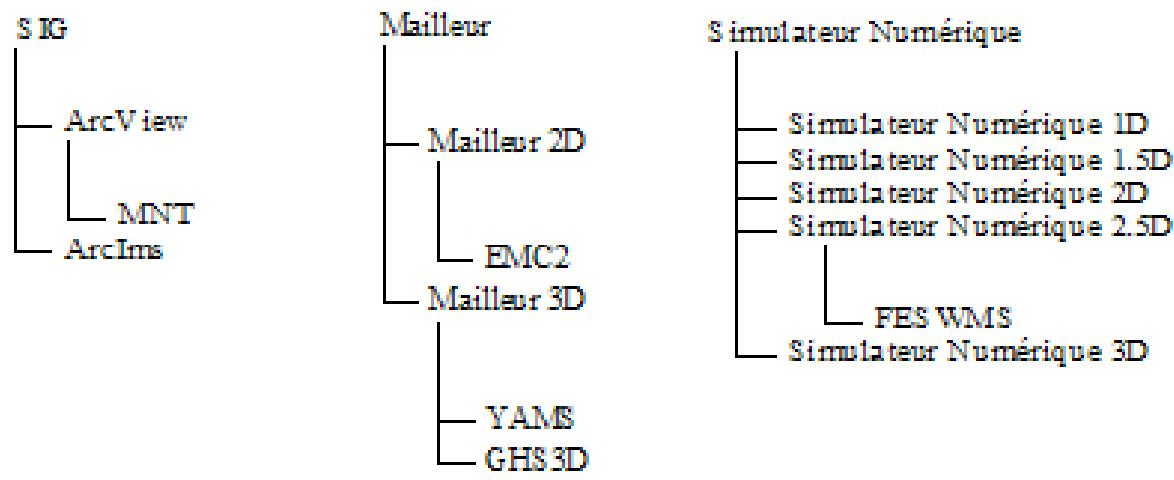

Figure 3. Ontologie préliminaire du domaine

numériques utilisées mais ne gardera qu'un seul niveau pour les deux entrées SIG et Mailleur.

\subsubsection{Profil utilisateur}

Un système adaptatif, comme son nom l'indique, est destiné à guider l'utilisateur et lui fournir l'information pertinente. En fait, il devrait idéalement, permettre à l'utilisateur de récupérer de l'information à partir des données auxquelles a accès le système. De plus, on note que le niveau d'interaction sera différent en fonction de l'utilisateur : s'il a un profil d'expert ou s'il est un utilisateur novice, avec une faible connaissance de la tâche à réaliser ou s'il est incapable de se servir d'une souris, etc. Dans cette optique, nous distinguons les utilisateurs, pour lesquels il est souhaitable de profiler les capacités cognitives, la compétence dans le domaine d'application du système et les contraintes sur leur mode d'interaction avec le système [18]. En général, un profil d'utilisateur rassemble l'ensemble des personnes ayant le même comportement (rôle) vis à vis du système. Dans ce sens, il serait intéressant aussi de bien identifier les catégories des personnes qui expriment les besoins en terme de fonctionnalités du système. Nous recenserons, pour chaque profil d'utilisateur, les caractéristiques, l'environnement de travail et les tâches potentielles des différents utilisateurs. A titre d'exemple, l'inventaire des informations requises pour les différents profils d'utilisateurs est présenté dans la figure (3.2.2) ci-dessous :

ARIMA - numéro spécial TAM TAM'05 
1. Caractéristiques de l'utilisateur

1.1. Attributs physiques

1.1.1. Age

1.1.2. Sexe

1.1.3. Limitations physiques

1.2. Connaissances

1.2.1. Expérience avec le système ou équivalent

1.2.2. Expérience avec l'ordinateur

1.2.2.1. Système opératoires maitrisés

1.2.2.2. Langages de programmation maitrisés

1.2.2.3. Outils maitrisés

1.2.3. Qualification

1.2.4. Fonction

1.2.5. Domaines d'application

1.2.6. Langues

2. Environnement de travail

2.1. Localisation // l'utilisateur travaille soit en équipe dans un labo ou centre de calcul soit seul sur son poste (bureau)

2.2. Usage // professionnel ou privé

2.3. Matériel

2.3.1. Matériel basique // PC, Station de travail, etc.

2.3.2. Périphériques locaux // imprimante, scanner, modem, etc.

2.3.3. Réseau // local, public, haut débit, etc.

2.4. Logiciels

2.4.1. Systèmes opératoires disponibles

2.4.2. Logiciels disponibles

3. Tâches potentielles

3.1. Liste des tâches // les plus utilisées

3.2. Caractéristiques des tâches

3.2.1. Objectif

3.2.2. Fréquence

3.2.3. Durée

3.2.4. Flexibilité // ordre des tâches

3.2.5. Connaissances requises

3.2.6. Ressources requises

Figure 4. Exemple d'inventaire

\section{Réalisation et applications (Application inondation)}

Le S2IW met à la disposition de l'utilisateur un ensemble d'outils, interconnectés entre eux par des interfaces intuitives et conviviales, pour la simulation numérique et la gestion des ressources hydriques : Eutrophisation, Inondations et Bassin Versant. A titre d'illustration, on se limite dans ce travail à présenter un exemple de scénario d'une simulation de crue [16]. Le S2IW permet ainsi, la génération d'un maillage du domaine de l'écoulement via le SIG ou EMC2 (Figure 5), l'exécution du simulateur numérique à savoir le code FESWMS ou SAINTVENANT (Figure 6) et la visualisation des résultats via SMS ou VISU (Figure 7). 


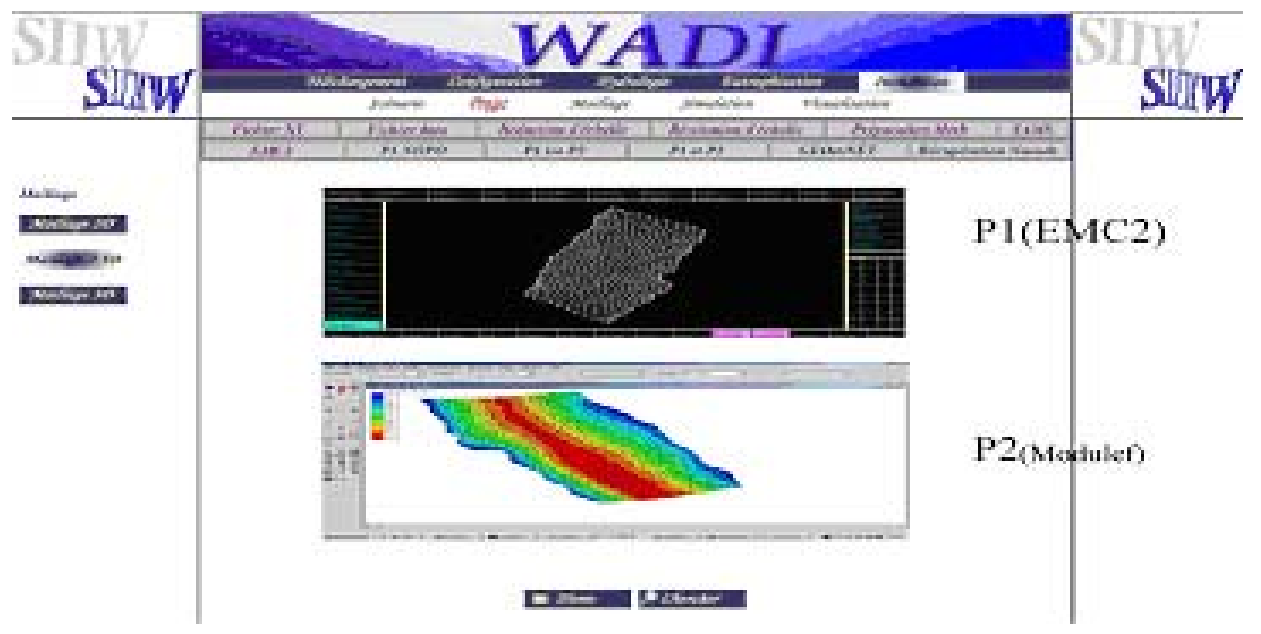

Figure 5. Maillage du cours d'eau

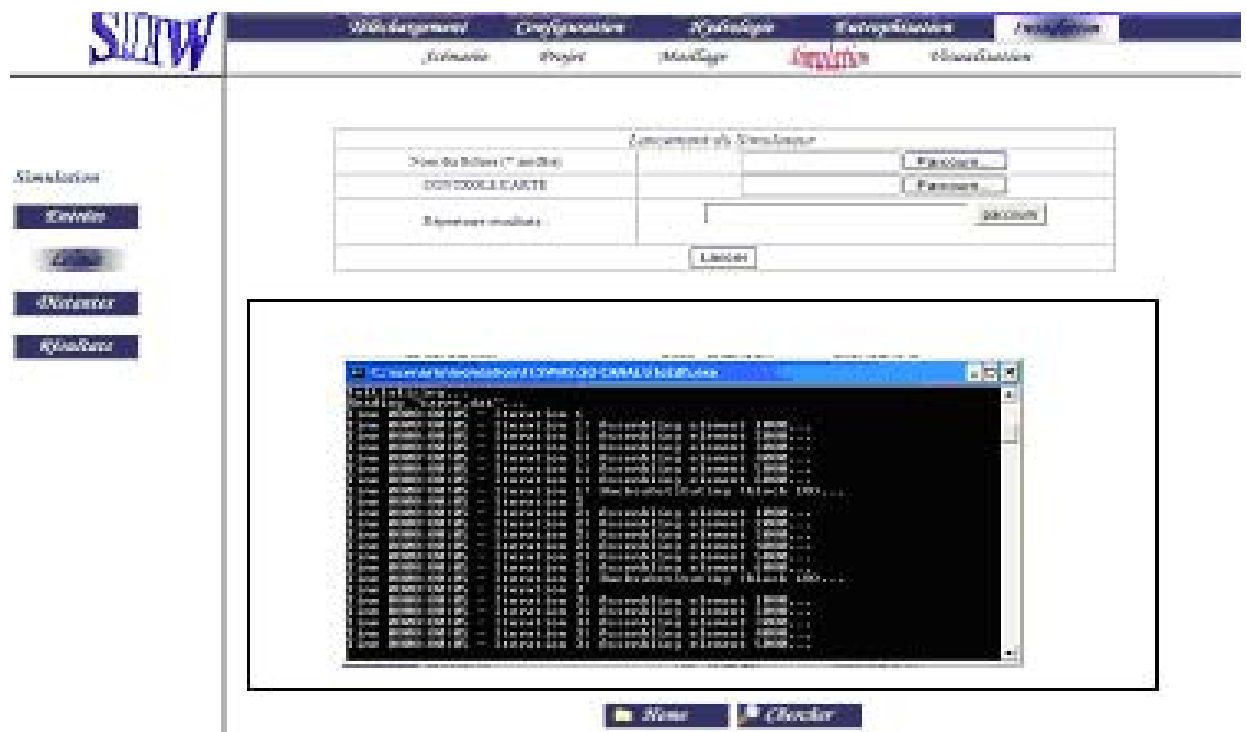

Figure 6. Lancement de la simulation 


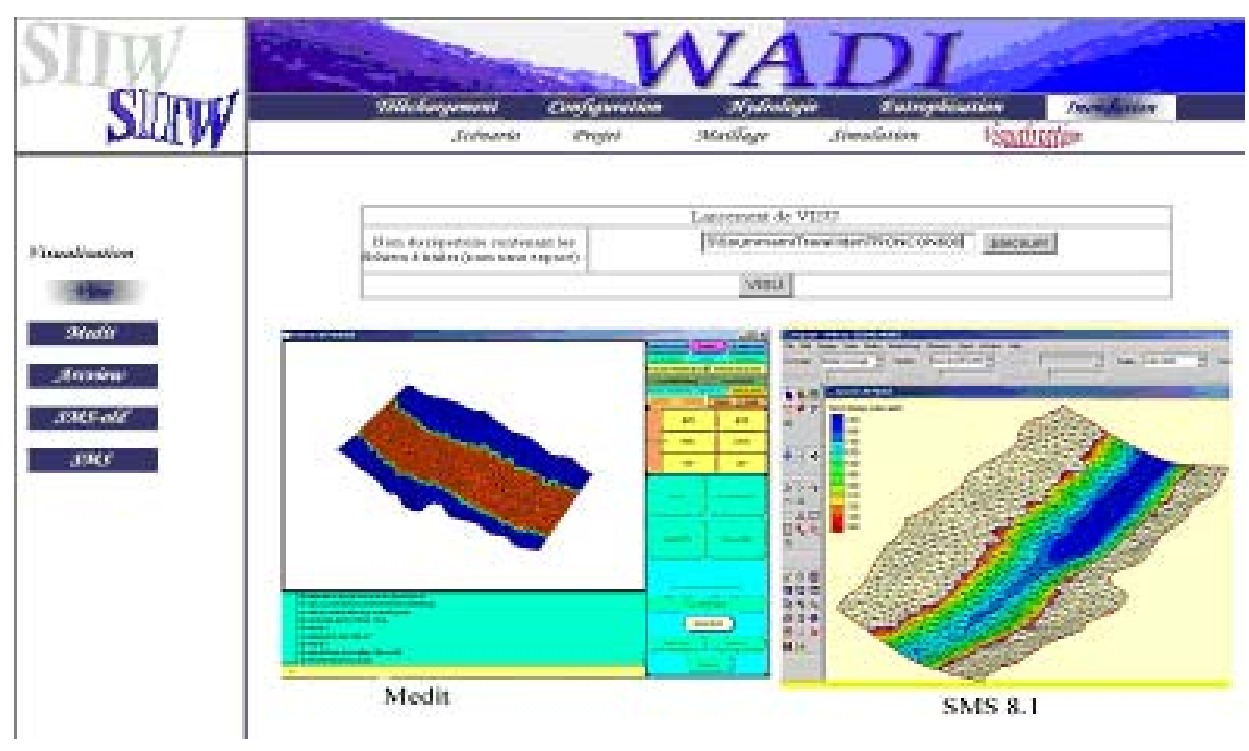

Figure 7. Visualisation des résultats sous SMS et VISU

\section{Conclusion}

Cette analyse préliminaire et détaillée de l'état de l'art sur l'adaptation et la personalisation en fonction de l'utilisateur a été amorcée afin d'étendre le S2IW vers le S2IAW (Système d'Information Intégré Adapté sous Web). Ce S2IAW sera un élément d'aide dans la chaîne de prise de décision permettant la simulation numérique, le traitement d'images et la gestion des données hétérogènes pour la prédiction et l'estimation de l'évaluation et de l'évolution des phénomènes hydriques.

De plus, une telle plate forme de développement, élargit son cercle d'utilisation et augmente l'échange des données et de résultats et par conséquent ajouter une nouvelle dimension en matière de recherche et de développement dans le domaine de l'ingénierie de l'eau. Par ailleurs, le S2IAW est tout à fait générique, sa transposition vers d'autres domaines d'application, voisins ou connexes, est relativement aisée : l'aménagement côtier (ressources marines, etc..), la gestion et la modélisation des ressources énergétiques, la qualité de l'air, la météorologie. 


\section{Bibliographie}

[1] Abdelwahed M., Dabaghi F., Two Proposed Models for the Numerical Study of the Treatment of Lake Eutrophication through Mechanical Aeration, Proceeding of the IASTED International Conference on Applied Simulation and Modelling (ASM 2004), pp. 148-153, Rhodes-Greece, Jun 2004.

[2] Abdelwahed M., Dabaghi F., Ouazar D., An alternative two phase flow model based on corrected water phase flow model for the simulation of eutrophised lake treatement by aeration process, International Conference on Thermal Engineering: Theory and Applications, ICTEA-HT4-04, Beirut-Lebanon, May-Jun 2004.

[3] Aussenac-Gilles N., BiÉBow B., Szulman S., Corpus analysis for conceptual modelling, Proceeding of EKAW, pp. 13-20, 2000.

[4] Bachimont B., Modélisation linguistique et modélisation logique des ontologies : l'apport de l'ontologie formelle, Actes d'IC, pp. 349-368, 2001.

[5] Barry C., Cormier C., Kassel G., Nobécourt J., Evaluation de langages opérationnels de représentation d'ontologies, Actes d'IC, pp. 309-327, 2001.

[6] BECHCHI M., Réalisation et implémentation du SR2I dans un Système d'Information Intégré sous Web WADI (S2IW)-Application aux ressources hydriques, Mémoire du Master Recherche Informatique, INRIA-EMI-Univ.Paris11, Sept 2004.

[7] Brusilovsky P., Adaptive Hypermedia, User Modeling and User-Adapted Interaction, 2001

[8] Dabaghi F., Henine H., Bechehi M., Rizk A., Gharbi A., Planning and Management Tools, report D5.3 WADI EC project, July 2004.

[9] Dabaghi F., Henine H., Bechchi M., Rizk A., Gharbi A., Web site, report D6.1 WADI EC project, July 2004.

[10] Dabaghi F., El Kacimi A., Nakhlé B., Numerical Analysis of a Priori Error Estimates for the Characteristics Mixed Finite Element Approximation of Shallow Water Equations, Proceeding of the IASTED International Conference on Applied Simulation and Modelling (ASM 2004), pp. 275-280, Rhodes-Greece, Jun 2004.

[11] Dabaghi F., El Kacimi A., Nakhlé B., Characteristics Finite Element Method for Shallow Water Equations, International Conference on Thermal Engineering: Theory and Applications, ICTEA-ES2-O2, Beirut-Lebanon, May-Jun 2004.

[12] Dabaghi F., El Kacimi A., Nakhlé B., A Priori Error Analysis of the Characteristics Mixed Finite Element Method for Shallow Water Equation, Proceeding of the 22nd IASTED International Conference on Modelling, Identification, and Control, (MIC 2003), pp. 459-464, Innsbruck-Austria, Feb 2003.

[13] Dabaghi F., Ouazar D., Prastacos P., Integrated Information System for Modeling and Management of Water Resources: Concept and Architecture, J. of Systems Analysis Modelling Simulation - SAMS, 2001.

[14] ESRI, ArcView, GIS The Geographic Information System for Everyone, 1995.

[15] HEC, Hydrologic Engineering Center-Hydrologic Modelling System HEC-HMSM, Technical Reference Manual, Mar 2000. 
[16] Henine H., Interface modèle hydrologique modèle Hydrodynamique au sein d'un Système d'Information Intégré sous Web S2IW incluant les SIG, Thèse de Magistère, INRIA-ENP, Dec 2004.

[17] IDER K., Modélisation hydrodynamique d'un cours d'eau. Application à l'Oued Soummam, Thèse de Magistère, INRIA-ENP, Juin 2004.

[18] Kobsa A., Koenemann J., Pohl W., Personalized hypermedia presentation techniques for improving online customer relationships, German National Research Center for Information Technology, 1999.

[19] LAME G., Knowledge acquisition from texts towards an ontology of French law, Proceedings of EKAW, pp. 53-62, 2000.

[20] Nobécourt J., A method to build formal ontologies from texts, Proceedings of EKAW, pp. 21-27, 2000.

[21] Souissi N., Dabaghi F., Integration of heterogeneous data and tools for monitoring and forecasting hydrous phenomena and processes, Proceedings of the 5th IFIPInternational Symposium on Environmental Software Systems (IFIP-ISESS 2003), pp. 253-261, ISBN 3-901882-16-2., May 2003.

[22] Souissi N., Dabaghi F., Ouazar D., Water resources modelling and simulation software: an integrated approach, Proc. of the 21 st IASTED International Conference on Applied Informatics, (AI 2003), pp. 913-918, Feb 2003.

[23] Talamali S., L'hydrodynamique des rivières et les systèmes d'information géographique, Thèse de Magistère, INRIA-ENP, Oct 2002.

[24] TomcAT, http://www.java.sun.com : Ressources JAVA.

[25] Vassileva J., A Task Centered Approach for User Modelling in Hypermedia Office Documentation System, User Modelling and User-Adapted Intercation, vol 6, $n$ ㅇ-3, pp. 185-224, 1996. 